\title{
Theoretische Überlegungen zur Modellierung und Erforschung von integrativem Fach- und Sprachenlernen
}

Jun.-Prof. ${ }^{\text {in }}$ Dr. ${ }^{\text {in }}$ Lena Heine

Seminar für Sprachlehrforschung, Fakultät für Philologie

Ruhr-Universität Bochum

\section{Einleitung}

Wie die Kompetenzmodelle der Schulfächer (für Österreich z.B. Bundesinstitut bifie 2011; für Deutschland Bildungsstandards, KMK 2015) verdeutlichen, wird die Rolle, die sprachliche Kompetenzen als Teil von Fachlichkeit spielen, mittlerweile in allen Fächern erkannt. Allerdings ist noch weitgehend unklar, was in diesem Zusammenhang konkret in die Aufgabenbereiche der einzelnen Didaktiken fällt. Hier stehen die Fächer vor bedeutenden Herausforderungen, die nicht nur die Erarbeitung des neuen Feldes „Sprache im Fach“ betreffen, sondern auch tief in das fachliche Selbstverständnis hineinreichen. Ganz konkret zeigt sich dies in unterrichtsbezogenen Entscheidungsfragen: Wie viel Zeit soll und kann im Sachfachunterricht in Sprachbildung bzw. -förderung investiert werden, ohne die eigentlichen Ziele des Sachfachs zu unterlaufen? Wann und wie sollen die FachlehrerInnen sprachliche Fehler der SchülerInnen korrigieren? Müssen SchülerInnen gezielt dazu gebracht werden, Sprache zu produzieren, oder ist davon auszugehen, dass ein reichhaltiger Input mit Fokus auf Inhalt genügt, um sich sprachlich weiterzuentwickeln? Muss für eine optimale fachintegrierte Sprachförderung in jede fachliche Aktivität ein Fokus auf Sprache eingebettet werden? Sollte (fehlende) sprachliche Kompetenz in die Benotung des Sachfaches einfließen - und wenn ja, mit welcher Begründung? 
Eine wissenschaftlich fundierte Beantwortung derartiger praxisorientierter Fragen erfordert zunächst eine grundlegende Vorstellung davon, was fachliches Lernen und was sprachliches Lernen eigentlich sind und wie beides miteinander in Beziehung steht. Schließlich stellt sich die Frage, wie sich aus fachlicher Sicht motivieren lässt, warum integrative Spracharbeit geleistet werden sollte. Stellt etwa der Sachfachunterricht lediglich eine gute Gelegenheit dar, sprachliche Kompetenzen weiterzuentwickeln, oder interagiert Sprache möglicherweise so mit fachlich-konzeptuellen Denkprozessen, dass über Spracharbeit auch ein Zuwachs an genuin fachlichen Kompetenzen angestrebt werden kann?

Dass fachliche und sprachliche Kompetenzen miteinander verbunden sind, steht spätestens seit den PISA-Studien außer Frage; ob es sich aber um eine kausale Beziehung handelt, bei der z.B. unausgereifte bildungssprachliche Fähigkeiten ursächlich für mangelhafte Leistungen in fachlicher Hinsicht sind, ist bislang noch nicht wirklich geklärt. Letztlich bedeutet ein Erschließen dieses Bereichs, dass das Verhältnis zwischen Denken und Sprechen in den Blick genommen wird und in Bezug auf die Spezifika von fachlichem Denken und schulsprachlicher Registerentwicklung (u.a. unter der Bedingung von Mehrsprachigkeit) ausgearbeitet werden muss. Erst eine solche theoretisch stringente und empirisch gestützte Vorstellung über diese Wechselbeziehung ermöglicht es, Fragen über den Zusammenhang zwischen Fach- und Sprachlernen hypothesentestend zu überprüfen und in zielgerichteter Weise zu antizipieren, wie der integrative Aufbau von Fach- und Sprachkompetenzen gesteuert werden kann. Eine Beschäftigung mit den theoretischen Grundlagen von integriertem Fach- und Sprachlernen wird im wissenschaftlichen Feld bislang noch kaum vorgenommen, somit bauen die didaktischen Überlegungen in diesem Bereich nur selten auf empirischen Ergebnissen auf. Der Schwerpunkt des folgenden Beitrags soll daher auf der Reflexion der Frage liegen, welche Voraussetzungen theoretischer Art vorliegen, um das komplexe Verhältnis zwischen fachlichen und sprachlichen Kompetenzen zu erhellen.

Ich möchte zur Begründung der Relevanz dieser Frage zunächst problematisieren, dass eine Spracharbeit im Fach, die vor allem durch sprachdidaktische Perspektivierungen geleitet ist, nicht wirklich integrativ ist. Vielmehr läuft sie aus fachlicher Sicht Gefahr, als Fremdleistung für ein fachfremdes Anliegen -

Barbara Hinger (Hg.), Zweite „Tagung der Fachdidaktik“ 2015.

Sprachsensibler Sach-Fach-Unterricht - Sprachen im Sprachunterricht.

(C) 2016 innsbruck university press, ISBN 978-3-903122-51-2, DOI 10.15203/3122-51-2 
der Förderung sprachlicher Kompetenzen - wahrgenommen zu werden. Danach möchte ich die bisher im Diskurs dominanten Theorierahmen daraufhin besprechen, wie sie sich für ein Detailverständnis des Zusammenhangs zwischen sprachlichem und nichtsprachlichem Wissensaufbau eignen. In diesem Zusammenhang sollen kognitiv-linguistische Ansätze als wichtige Kandidaten für eine integrative Theorie auf Mikroebene vorgeschlagen werden, die auf Mesoebene mit soziofunktionalen Theorieansätzen kompatibel ist. Abschließend möchte ich illustrieren, wie aus der Kenntnis der theoretischen Prämissen empirisch überprüfbare Forschungsfragen gestellt werden können.

\section{Grenzen der Zweitsprachendidaktik in der Übertragung auf sachfachliche Kontexte}

Die Fragen, wie man sprachlichen Kompetenzaufbau anleiten und in welcher Art von Unterrichtssetting man ihn optimieren kann, werden bekanntlich in erster Linie in den Erst-, Zweit- und Fremdsprachendidaktiken bearbeitet. Es ist davon auszugehen, dass ein auf Kommunikationsfähigkeit und Handlungskompetenz ausgerichteter Sprachunterricht für das Sprachenlernen sinnvoller ist als ein rein formfokussierender Unterricht (z.B. Long \& Robinson 1998; zusammenfassend Ellis \& Shintani 2014; s. für aktuellen Überblick mit DaZ-Fokus Rotter 2015). Aufgabenbasierte Lernsettings mit einem Fokus auf Inhalt - Lernsituationen im Sachfachunterricht durchaus ähnlich - sind den Sprachdidaktiken also vertraut. Viele der eingangs gestellten unterrichtspraktischen Fragen zum Umgang mit Sprache im Fach (bzgl. Fehlerkorrektur, der Relevanz von Output etc.) müssten sich damit auf der Basis des Kenntnisstandes der Sprachdidaktiken beantworten lassen. Budde \& Michalak (2015: 9) formulieren dies wie folgt: ,[Es] sind [...] die Sprachenfächer [...], auf die jedes Fach schauen sollte: Ihre Zielsetzungen, die zu vermittelnden Kompetenzen und ihre dafür eingesetzten Methoden des binnendifferenzierenden und selbstgesteuerten Unterrichts könnten eine Orientierung und zugleich eine Basis für den Erwerb von Fähigkeiten darstellen, die für das sprachliche Lernen in den Fächern zu nutzen sind.“

Barbara Hinger (Hg.), Zweite „Tagung der Fachdidaktik“ 2015.

Sprachsensibler Sach-Fach-Unterricht - Sprachen im Sprachunterricht.

(C) 2016 innsbruck university press, ISBN 978-3-903122-51-2, DOI 10.15203/3122-51-2 
Wenn dieser Argumentation auch eine grundlegende Plausibilität innewohnt, so ist sie doch aus mehreren Gründen kritisch zu sehen: Zum einen impliziert sie, dass die Sprachdidaktiken bereits die Antworten für eine wirkungsvolle Sprachförderung im Fach haben und sich die Sachfachdidaktiken sich nun von den SprachexpertInnen sozusagen anlernen lassen und eine Art Zusatzidentität als SprachlehrerInnen aufbauen müssten. Lehrkräfte in den Sachfächern sollen aber nicht zusätzlich zu ExpertInnen im Zweit- oder sonstigem Spracherwerb werden. Sie sollen Sachfachlehrkräfte sein, die die sprachlichen Anforderungen ihres Faches überblicken, sie adaptieren können und deren Funktion für das Erreichen fachlicher Ziele verstehen und an SchülerInnen vermitteln können, dabei jedoch klar ihre Sachfachziele im Blick behalten.

Weiterhin bedeutet eine einseitig mit Spracherwerbszielen begründete integrative Sprachförderung, dass erhebliche Anteile des Fachunterrichts für nicht im Kern sachfachdidaktische Aktivitäten bereitgestellt werden müssten. Geht die Argumentation so: Sprachförderung muss integrativ in allen Fächern stattfinden, nicht, weil die Fächer davon profitieren, sondern weil dies am optimalsten für die Entwicklung von sprachlichen Kompetenzen ist - könnte dies bedeuten, dass die Fächer die Aufgabe der Sprachförderung nicht als ihre eigene sehen. Fachintegrierte Spracharbeit könnte damit Gefahr laufen, als eine Fremdleistung betrachtet zu werden, der angesichts sowieso schon begrenzter Ressourcen nur wenig Aufmerksamkeit gewidmet wird.

Zusätzlich führt eine einseitig sprachdidaktische Begründung von fachintegrierter Sprachförderung auf theoretischer Seite dazu, dass die integrative Natur von Fach- und Sprachenlernen nicht in den Blick genommen, damit nicht präzise erforschbar ist und somit auch nicht systematisch didaktisch nutzbar wird.

In diesem Zusammenhang lautet mein erstes Plädoyer, gezielt aus der Fächerperspektive mit ihren genuin fachspezifischen Interessen einen Zugang zum Thema Sprachförderung zu suchen. Eine Frage in diesem Zusammenhang wäre, welche Vorteile ein Fokus auf Sprache für das fachliche Lernen haben könnte. Damit würde fachintegrierte Spracharbeit primär fachlich motiviert und als Mehrwert für das jeweilige Schulfach erfasst, was nicht zuletzt in Hinblick auf die begrenzten Ressourcen, die in der LehrerInnenbildung, aber auch im Unterrichtsalltag für diesen Bereich freigemacht werden können, sinnvoll erscheint.

Barbara Hinger (Hg.), Zweite „Tagung der Fachdidaktik“ 2015.

Sprachsensibler Sach-Fach-Unterricht - Sprachen im Sprachunterricht.

(C) 2016 innsbruck university press, ISBN 978-3-903122-51-2, DOI 10.15203/3122-51-2 
Um diesen Fragenkomplex wissenschaftlich fundiert bearbeiten zu können, bedarf es zunächst eines Theorierahmens, der eine Modellierung sowohl der fachlichen wie auch der sprachlichen Dimensionen ermöglicht. Hier lohnt ein Blick darauf, ob die bislang im Diskurs zum integrativen Fach- und Sprachlernen vorzufindenden Theorien eine solche integrative Modellierung erlauben bzw. wo ihre Begrenzungen liegen. Dies soll im folgenden Abschnitt genauer beleuchtet werden.

\section{Forschungsdiskurse im Bereich integrativen Fach- und Sprachlernens}

Es lassen sich verschiedene Teilgebiete identifizieren, die sich bislang mit Aspekten des integrativen Fach- und Sprachlernens befasst haben.

Im Fokus der Aufmerksamkeit ist hier momentan das Feld der Zweitsprachenerwerbsforschung im Kontext von Deutsch als Zweitsprache (DaZ), dem der aktuelle bildungspolitisch ausgerichtete deutschsprachige Diskurs zur Förderung von SchülerInnen aus bildungsfernen Schichten mit besonderem Augenmerk auf LernerInnen mit Zuwanderungsgeschichte zuzuordnen ist. Aktuelle Ansätze folgen hierbei klar einer integrativ in allen Fächern verankerten Stoßrichtung, auch wenn sich dies in der LehrerInnenausbildung noch nicht flächendeckend niederschlägt (vgl. für einen Überblick über die Situation in Deutschland Baumann \& Becker-Mrotzek 2014).

Ein verwandtes Arbeitsfeld stellt der Bilinguale Sachfachunterricht (BU) dar (vgl. für einen Überblick Breidbach \& Viebrock 2012). Wie im aktuellen DaZDiskurs geht es hier um die Beantwortung der Frage, wie ein Sachfachunterricht gestaltet werden kann, in dem die sachfachlichen Kompetenzen im Medium einer Fremd- bzw. Zweitsprache aufgebaut und damit gleichzeitig L2-Kompetenzen vorangetrieben werden können.

Ebenfalls eine deutliche fachlich-inhaltliche Dimension weist der im deutschsprachigen Bereich nur randständig beforschte hochschulische Fremdsprachenunterricht auf. So beinhaltet ein großer Teil hochschulspezifischer Fremdspra-

Barbara Hinger (Hg.), Zweite „Tagung der Fachdidaktik“ 2015.

Sprachsensibler Sach-Fach-Unterricht - Sprachen im Sprachunterricht.

(C) 2016 innsbruck university press, ISBN 978-3-903122-51-2, DOI 10.15203/3122-51-2 
chenkurse wie „Englisch für Biologen“ oder „Deutsch für ausländische Studierende im Studiengang Architektur" eine klar fachbezogene Dimension, sodass der hochschulische Fremdsprachenunterricht stark an den Bedarfen, die der fachlichen Perspektive entspringen, ausgerichtet werden muss (z.B. Voss 2012).

Obwohl all diese Bereiche an der Schnittstelle von Fach und Sprache liegen, sind sie bislang auf wissenschaftlicher Basis vorrangig aus sprachdidaktischer Perspektive bearbeitet worden. So wird der Sprachförder-/DaZ-Diskurs vor allem durch Forschende mit germanistischem (aber auch erziehungswissenschaftlichem) Hintergrund dominiert; DidaktikerInnen aus den Sachfächern bilden klar die Ausnahme, wenn es auch wichtige Ansätze aus den Fächern gibt (z.B. für Geschichte Handro \& Schönemann 2010; für Mathematik z.B. Prediger 2013; für Chemie z.B. Busch \& Ralle 2013). Auch der BU-Ansatz wird von der Fremdsprachenforschung dominiert, obwohl in der Unterrichtspraxis im deutschsprachigen Bereich fachliche Lernziele über sprachliche gestellt werden (Heine i.Dr.). Die Fremdsprachenforschung überprüft etwa, ob der Bilinguale Sachfachunterricht ein optimaleres Setting für den Erwerb einer Fremdsprache darstellt als der herkömmliche Fremdsprachenunterricht. Wenige Studien beziehen sich auf den BU in seiner Qualität als Sachfachunterricht, gemessen am Regelunterricht (s. Überblick in Heine 2013). Versuche, das integrative Moment dieser Unterrichtsform systematisch zu untersuchen, gibt es bislang nur vereinzelt (z.B. Bonnet 2004, Heine 2010; s. für einen wichtigen theoretischen Ansatz in Bezug auf fachliche Diskursfunktionen Dalton-Puffer 2013).

Auch der im deutschsprachigen im Vergleich zum anglophonen Raum nur sehr marginal beforschte Bereich des hochschulischen Fremdsprachenunterrichts ist in der Forschungscommunity klar als Fremdsprachenunterricht - nicht als integrativer Fach- und Fremdsprachenunterricht - identifiziert und wird innerhalb der Methoden und Zugangsweisen der Fremdsprachenforschung bearbeitet, auch wenn die fachliche Komponente als wichtig erkannt wird. Hier kommt im Übrigen hinzu, dass es im Vergleich zum schulischen Feld keine nennenswerte Hochschuldidaktik der einzelnen Fächer gibt, die sich gezielt mit der Vermittlung von hochschulischen fachlichen Kompetenzen auseinandersetzen würde und eine Folie bereitstellen könnte, vor der Kompetenzdimensionen eines Fachstudiums erfassbar gemacht werden würden.

Barbara Hinger (Hg.), Zweite „Tagung der Fachdidaktik“ 2015.

Sprachsensibler Sach-Fach-Unterricht - Sprachen im Sprachunterricht.

(C) 2016 innsbruck university press, ISBN 978-3-903122-51-2, DOI 10.15203/3122-51-2 


\section{Dominante Theorierahmen in den genannten Forschungsdiskursen}

Sowohl DaZ, BU, aber auch der hochschulische Fremdsprachenunterricht operieren in ihren aktuellen europäischen Diskursen dominant mit einer Auffassung von Spracherwerb, die weniger durch einen Detailblick auf sprachliche Strukturen geprägt ist, sondern die sprachliche Funktion in Interaktionssituationen und Sprache somit in ihrer sozialen und kulturellen Geprägtheit betont; diese Auffassung korrespondiert deutlich mit dem Sprachverständnis, das sich im Europäischen Referenzrahmen für Sprachen manifestiert. Sprachliche Normen unterliegen keinem in sich geschlossenen Regelwerk, sondern bilden sich durch wiederkehrende Verwendungssituationen heraus. Sprache ist nach diesem Verständnis also kein System aus Elementen und Regeln ihrer Kombinierbarkeit, das es gilt, möglichst vollständig und fehlerfrei zu erwerben. Vielmehr wird Sprache als Werkzeug metaphorisiert, mit dessen Beherrschung es möglich wird, Zugang zu Gemeinschaften zu erhalten und kommunikativ-soziale Ziele zu erreichen. Sprachliche Struktur ist damit nicht entweder, richtig' oder ,falsch', sondern mehr oder weniger ,kommunikativ adäquat' und mehr oder weniger ,in der Diskursgemeinschaft akzeptiert'.

Besonders weit verbreitet sind im sprachendidaktisch orientierten DaZ- und BU-Diskurs Ansätze, die soziale und kulturelle Aspekte von Sprache betonen (vgl. die stark kulturorientierten BU-Modelle von Hallet 1998; Breidbach 2007; Coyle, Hood \& Marsh 2010; Zydatiß 2007: 16). Sie spiegeln generell das in der Fremd- und Zweitsprachenforschung derzeit dominante Paradigma wider, das insbesondere durch die Soziokulturelle Theorie nach Wygotski beeinflusst ist (ein- und weiterführend z.B. Lantolf, Thorne \& Poehner 2015; Lantolf \& Poehner 2014). Makrotheoretisch ist das hier enthaltene Sprachverständnis den soziokognitiv orientierten Sprachsozialisationstheorien zuzuordnen (vgl. Duff \& Talmy 2011). Die Soziokulturelle Theorie stellt keine Sprachtheorie im eigentlichen Sinne dar, sondern eine Sozialisations- und Entwicklungstheorie, in der Sprache aber eine zentrale Rolle eingeräumt wird. Sie geht bekanntlich davon aus, dass Kompetenzentwicklung primär durch soziale Interaktionen angestoßen wird und

Barbara Hinger (Hg.), Zweite „Tagung der Fachdidaktik“ 2015.

Sprachsensibler Sach-Fach-Unterricht - Sprachen im Sprachunterricht.

(C) 2016 innsbruck university press, ISBN 978-3-903122-51-2, DOI 10.15203/3122-51-2 
dass Menschen ihre mentalen Funktionen mit Hilfe verschiedener Stimuli kontrollieren und regulieren - diese können einfacher Natur sein, etwa ein Knoten im Taschentuch als Erinnerungshilfe, oder komplexer, etwa Symbole und Zahlen oder Diagramme als Hilfen für die Konstruktion einer komplexen Anlage. Sprache als besonders elaboriertes semiotisches System spielt nach soziokulturellem Verständnis eine zentrale Rolle: Mit Hilfe von Sprache als bedeutungskommunizierendem System sind Menschen in der Lage, ihre eigenen Kognitionen zu regulieren. Sprache bietet damit die Grundlage, um komplexere kognitive Fähigkeiten herauszubilden.

Die im germanistisch geprägten DaZ-Diskurs oft verwendete Funktionale Pragmatik (z.B. Ehlich \& Rehbein 1983) versteht sich demgegenüber in erster Linie als Sprachtheorie, die Diskurse in verschiedenen sozialen (insbesondere institutionalisierten) Kontexten beschreibt, und erst nachgeordnet auf dieser Grundlage einen didaktischen Blick eröffnet. Wie die Soziokulturelle Theorie geht sie davon aus, dass die standardmäßig auftretenden kommunikativen Anforderungen in institutionellen Zusammenhängen wie etwa der Schule zu einer Grundlage für die Einschätzung davon führen, welche sprachlichen Muster als interinstitutionell zulässig und anerkannt akzeptiert werden und welche nicht. Ganz ähnliche Ansätze finden sich in den anglophon ausgerichteten Teilbereichen des hochschulischen (Zweit-/Fremd-)Sprachenunterrichts in Gestalt der Systemic Functional Linguistics (Halliday \& Martin 1993; Halliday \& Matthiessen 2004), die v.a. im Bereich English for Academic Purposes (EAP) mit dem GenreAnsatz (z.B. Bazerman 2009; Schleppegrell 2004) verwendet wird. Auch sie basiert auf einer soziokulturellen Sicht, wobei EAP im Sinne der Systemic Functional Linguistics eine stärkere Fokussierung auf sprachliche Analyse legt, um die Eigenheiten fachlicher Diskurse zu erfassen und diese wiederum an LernerInnen vermitteln zu können. Die parallele Strömung der Academic-Literacy-Forschung (z.B. Lillis \& Scott 2008; Thesen \& Pletzen 2006) vertritt eine eher ethnographischkritische Perspektive und fokussiert weniger Sprach- und Textanalysen als die Beschreibung von Diskurspraktiken in fachlichen Zusammenhängen (vgl. Coffin \& Donohue 2012).

Dieser kurze Überblick skizziert, dass die dominanten Theorien, die im Überschneidungsbereich von Fach- und Sprachlernen derzeit zur Anwendung kom-

Barbara Hinger (Hg.), Zweite „Tagung der Fachdidaktik“ 2015.

Sprachsensibler Sach-Fach-Unterricht - Sprachen im Sprachunterricht.

(C) 2016 innsbruck university press, ISBN 978-3-903122-51-2, DOI 10.15203/3122-51-2 
men, grundlegend funktionaler Art sind, in einem soziolinguistisch und kulturgebundenen Verständnis basiert sind und Sprache als Werkzeug (kognitiver Art, als Mittel zur Teilhabe) metaphorisieren. Weiterhin deutet sich hier an, dass die soziale Interaktion als zentraler Faktor beim Sprachenlernen gesehen wird.

\section{Soziofunktionale Theorien und die Erforschung von integrativem Fach- und Sprachlernen}

Für den Kontext einer integrativen Sicht auf Fach- und Sprachlernen bieten die gerade kurz charakterisierten sozio-funktionalen Theorien grundsätzlich sinnvolle Theorierahmen, weil sie Sprache als wesentlichen Bestandteil von (institutionell-fachlicher) Sozialisation und sprachliche Normen als in (fachspezifischen) sozialen Diskursen erklärbar machen und damit sowohl eine sprachliche als auch eine fachliche Dimension anbieten.

Von Vorteil ist darüber hinaus, dass soziofunktionale Theorien nicht nur in Spracherwerbskontexten verwendet werden, sondern auch von einigen Sachfachdidaktiken, z.B. in der Biologie- (z.B. Krüger \& Vogt 2007) oder der Physikdidaktik (Kircher, Girwidz \& Häußler 2014: 459, 652). Innerhalb dieses theoretischen Rahmens lassen sich sachfachdidaktisch wichtige Aspekte des Aufbaus von konzeptuellem Wissen abbilden und die Veränderung von weniger elaborierten hin zu fachlich adäquaten Vorstellungen im Sinne eines Conceptual Change oder ähnlicher fachlicher Entwicklungskonstrukte erfassen.

Da zumindest die Soziokulturelle Theorie (SKT) in ihrer Ausrichtung auf kognitive und soziale Entwicklung von Kindern starke pädagogische Implikationen formuliert, bietet sie eine gute Grundlage für didaktische Forschung. Versteht man Sprache in soziokulturellem Verständnis als Mediationswerkzeug, mit dessen Hilfe nicht nur Gedankeninhalte versprachlicht, sondern insbesondere komplexe, abstrakte Zusammenhänge überhaupt erst kognitiv verankert werden können, in denen Sprache stellvertretend für sinnliche Erfahrbarkeit steht, so hat dies für die gezielte Erforschung des Zusammenhangs von Fach- und Sprachlernen unmittelbare Implikationen.

Barbara Hinger (Hg.), Zweite „Tagung der Fachdidaktik“ 2015.

Sprachsensibler Sach-Fach-Unterricht - Sprachen im Sprachunterricht.

(C) 2016 innsbruck university press, ISBN 978-3-903122-51-2, DOI 10.15203/3122-51-2 
Obwohl die Diskurse zum integrativen Fach- und Sprachlernen damit eine allgemein akzeptierte theoretische Verankerung aufweisen und eine gut vorbereitete Ausgangslage für hypothesentestende Forschung bieten, ist das Wechselverhältnis von Sprach- und Fachlernen bislang noch kaum Gegenstand empirisch basierter Ausarbeitung der damit einhergehenden theoretischen Grundlagen in der deutschsprachigen didaktischen Forschung gewesen. Einen Grund sehe ich zumindest für die Fremdsprachenforschung im generellen Mangel an theoretischer Durchdringung der Disziplin (vgl. Heine 2015). In der anglophonen Forschung ist die Situation etwas anders, wie z.B. Lantolf \& Poehner (2014) in ihrem Überblick über Forschung im Bereich der Fremdsprachenforschung, die sich auf soziokultureller Grundlage auch mit der Beziehung zum Inhaltslernen auseinandersetzt, zeigen.

Didaktisch relevante Annahmen über das Wechselverhältnis von Fach- und Sprachlernen, die stringent aus dem soziokulturellen Verständnis herleitbar und empirisch überprüfbar sind, wären etwa die folgenden:

\section{SKT-Forschungsfrage Beispiel 1}

Theoretisch motivierte Annahme: Die Soziokulturelle Theorie geht davon aus, dass Menschen insbesondere dann über ihre Umwelt lernen, wenn sie die Bedeutung von Artefakten erfassen, also verstehen, wie sie diese Artefakte nutzen können. Sprache fungiert dabei als besonders wichtiges Mediationsinstrument, das es z.B. erlaubt, auch abstrakte und nicht sinnlich erfahrbare Phänomene zu repräsentieren und damit erfahrbar zu machen.

Testbare Hypothese: LernerInnen, die mit sprachlichen Beschreibungen von Phänomenen vertraut gemacht werden, die für die LernerInnen die Aktivität ihrer Erstellung und ihres Einsatzgebietes implizieren, werden das Phänomen besser verstehen und anwenden können als LernerInnen, die mit einer sprachlichen Beschreibung arbeiten, die nur auf eine Liste von Eigenschaften des Phänomens ohne dessen strukturelle und funktionale Dimensionen rekurrieren (vgl. Davydov 2004: 82ff).

Barbara Hinger (Hg.), Zweite „Tagung der Fachdidaktik“ 2015.

Sprachsensibler Sach-Fach-Unterricht - Sprachen im Sprachunterricht.

(C) 2016 innsbruck university press, ISBN 978-3-903122-51-2, DOI 10.15203/3122-51-2 


\section{SKT-Forschungsfrage Beispiel 2}

Theoretisch motivierte Annahme: Die Soziokulturelle Theorie geht davon aus, dass Sprechen eine grundsätzlich an AdressatInnen (andere oder sich selbst) gerichtete Tätigkeit darstellt, und die Fähigkeit zur Verbalisierung von Tätigkeiten eine wichtige Voraussetzung für die Entwicklung vom konkreten zum abstrakten Denken darstellt, in der ausgehend von der Manipulation eines konkreten Gegenstands über eine Phase der Verbalisierung die Fähigkeit zur rein gedanklichen Aktivitäten erwächst (Wygotski 1987: Kap. 7).

Testbare Hypothese: LernerInnen, die sich selbst oder anderen LernerInnen fachliche Zusammenhänge erklären, indem sie diese selbstständig in sprachlicher Form zum Ausdruck bringen, erreichen die Fähigkeit, mit bestimmten abstrakten fachlichen Zusammenhängen umzugehen schneller als LernerInnen, die keine Versprachlichungsphase durchlaufen (vgl. Swain et al. 2009 mit ähnlichem Design).

\section{Ausschärfung soziofunktionaler Theorien auf theoretischer Mikroebene}

Soziofunktional ausgerichtete Ansätze zur Sprachverwendung in fachlichen Kontexten werden verschiedentlich der Kritik ausgesetzt, dass sie unspezifisch bezüglich ihrer theoretischen Verankerung auf der Mikroebene sind: Sie setzen auf vergleichsweise hohem funktionalem Abstraktionsniveau an und konkretisieren in der Regel nicht, welche Art von Wissensrepräsentation und Lernprozesse im Einzelnen anzunehmen und wie diese modellierbar sind (kritisch bezüglich der theoretischen Fundierung der hermeneutisch arbeitenden Funktionalen Pragmatik ist beispielsweise Weigand 1992; die fehlende Positionierung bezüglich der Repräsentationsebene der Systemic Functional Linguistics wird kritisch in Fawcett 2000: Kap .7 diskutiert, vgl. auch Butler 2013 zur linguistischen Basis der Soziokulturellen Theorie vgl. Lantolf \& Poehner 2014: 80ff.). Soziofunktionale Theorien spezifizieren also weder, wie sich die Entwicklung von Sprachwissen, 
noch die anderer - z.B. fachlicher - Wissensformen konkret vollzieht und wie man sich diese im Einzelnen vorstellen muss. Besteht Sprachwissen vorrangig aus unbewusstem Regelwissen oder aus memorierten Ein- und Mehrworteinheiten und Repräsentationen ihrer pragmatischen Funktionen? Ist Sprachlernen unmittelbar mit anderen Formen von Lernen verbunden, oder unterliegen beiden jeweils verschiedene Prozesse? Müssen folglich beide auf verschiedene Weisen angeleitet werden, oder entwickeln sie sich unter denselben Bedingungen?

Allerdings werden seit kurzem Vorschläge gemacht, wie diese theoretische Unschärfe aufgehoben werden kann. Butler (2013) etwa plädiert dafür, die Systemic Functional Linguistics durch die Erkenntnisse der Kognitiven Linguistik und der Psycholinguistik zu ergänzen, und auch Lantolf \& Poehner (2014: 80ff., 217ff.) identifizieren gezielt die Kognitive Linguistik als besonders geeignete Entsprechung der Soziokulturellen Theorie auf spracherwerbstheoretischer Mikroebene. Sie präsentieren erste Studien, die auf den Grundannahmen der Kognitiven Linguistik innerhalb eines soziokulturellen Paradigmas auf empirischer Basis hypothesentestende Forschung betreiben. Auch Dalton-Puffer (2013) befürwortet kognitiv-linguistische Ansätze zur Verwendung in integrativen Fach- und Sprachlernkontexten.

Wie ich an anderer Stelle bereits mit Fokus auf Theorien in der Fremdsprachenforschung genauer ausgeführt habe (Heine 2015), eignen sich kognitiv-linguistische Theorien (vgl. Überblick z.B. Evans \& Green 2006) deshalb für eine Verbindung mit soziofunktionalen Rahmungen, weil sie die wesentlichen Grundannahmen teilen, dabei aber auf unterschiedlichen Abstraktionsniveaus ansetzen: Beide Theoriefamilien gehen davon aus, dass sprachliches wie nichtsprachliches Wissen durch sinntragenden Gebrauch aufgebaut wird. Sprache erfüllt eine soziale Funktion und ist in ihrer Struktur durch die kulturellen Rahmungen prototypischer Verwendungssituationen geprägt. Daraus ergibt sich, dass sprachliche wie nichtsprachliche Wissensstrukturen nicht arbiträr sind, sondern Bedeutung und Funktion widerspiegeln. Die Kognitive Linguistik geht damit nicht davon aus, dass Sprachwissen grundsätzlich besondere kognitive Strukturen anlegt und in seiner Verarbeitung anderen Prinzipien gehorcht als andere Formen von Wissen; vielmehr stellt sie eine Spezifizierung einer allgemeinen Kognitionstheorie auf Sprachwissen dar. Wie andere Formen von Wissenserwerb wird der Aufbau

Barbara Hinger (Hg.), Zweite „Tagung der Fachdidaktik“ 2015.

Sprachsensibler Sach-Fach-Unterricht - Sprachen im Sprachunterricht.

(C) 2016 innsbruck university press, ISBN 978-3-903122-51-2, DOI 10.15203/3122-51-2 
von Sprachwissen dabei als probabilistischer Vorgang des Stärkens von mentalen Vernetzungsstrukturen innerhalb konnektionistischer Netzwerke modelliert, die in der Repräsentation prototypischer Strukturen und radialer Kategorien münden und denen konzeptuelle Metaphern als grundlegendes kognitives Prinzip unterliegen. Ein kognitiv-linguistisches Verständnis kann u.a. mit der Dynamik von Spracherwerb, der Verbundenheit von Sprache und kulturellem Wissen, der sozialen Bedeutung von Sprache im Sinne soziofunktionaler Theorien verknüpft werden; sprachliche Struktur ist nach beiden Sichtweisen auf allen sprachlichen Ebenen durch Bedeutung motiviert.

Die Kognitive Linguistik bietet als empirisch ausgerichtete Forschungsdisziplin bereits eine gut fundierte Grundlage, die es erlaubt, solide Aussagen über den Aufbau von Sprachwissensstrukturen und diesen beeinflussende Faktoren aufzustellen (z.B. Evans \& Green 2006). Bislang ist dieses Feld allerdings vorrangig mit sprachwissenschaftlich-theoretischem Interesse bearbeitet worden und findet in didaktischem Zusammenhang noch so gut wie keine Beachtung. Die Vorteile der Kognitiven Linguistik liegen aber auch für didaktische Forschung auf der Hand: Verknüpft man sozio-funktionale Theorien auf Makroebene mit der Kognitiven Linguistik auf Mikroebene, erhält man einen stimmigen Rahmen zur Erfassung der sozialen Situation von Unterricht mit seinen jeweils fachspezifischen diskursiven Anforderungen und seiner kulturellen Geprägtheit. Sie können gleichzeitig auf der Mikroebene mit klarem und empirisch untermauertem Verständnis von Prozessen des Erwerbs von Sprachwissen durch Gebrauch vor dem Hintergrund von kulturell geprägtem und individuell ausgeformtem Vorwissen verbunden werden, das direkt mit fachlichen Vorstellungen von Conceptual Change und Laienvorstellungen in Beziehung gesetzt werden kann.

\section{Kognitive Linguistik und die Erforschung integrativen Fach- und Sprachlernens}

Die inhärenten Prämissen der Kognitiven Linguistik (KL) lassen sich ebenfalls in überprüfbare Forschungsfragen überführen, in denen gezielt das integrative

Barbara Hinger (Hg.), Zweite „Tagung der Fachdidaktik“ 2015.

Sprachsensibler Sach-Fach-Unterricht - Sprachen im Sprachunterricht.

(C) 2016 innsbruck university press, ISBN 978-3-903122-51-2, DOI 10.15203/3122-51-2 
Moment von fachintegrierter Spracharbeit in den Blick genommen werden kann. Ich skizziere auch hierfür einige Beispiele:

\section{KL-Forschungsfrage Beispiel 1}

Theoretisch motivierte Annahme: Eine Grundannahme der Kognitiven Linguistik ist, dass sprachliche Struktur (sowohl auf Morphem-, Wort-, Phrasen-, Satz-, aber auch auf Diskursfunktions- und Textebene) nicht arbiträr ist, sondern funktional die mit ihrem Gebrauch verbundenen kognitiven Funktionen widerspiegelt. Das kognitiv-linguistische Paradigma ist so vereinbar mit der Annahme, dass spezifische Charakteristika von sachfachlichen Anforderungen an Sprache in funktionaler Verbindung zu den im Sachfachunterricht auszubildenden Kognitionen stehen. Wenn im Sachfachunterricht beispielsweise das Ziel angestrebt wird, im Sinne eines Conceptual Change den Fokus des Lernenden weg vom Einzelphänomen in der subjektiven Erfahrungswelt und hin zu einer Wahrnehmung in einem abstrahierten, objektivierten systemischen Ganzen zu vollziehen, dann hat diese gedankliche Aktivität eine Entsprechung auf der Ebene der damit assoziierten sprachlichen Form. Genauer: Alltagssprache und Bildungs- bzw. Fachsprache sind nicht nur unterschiedliche Ausdrucksformen für dieselben Inhalte, sondern verweisen auf verschiedenartige Denkeinheiten.

Überprüfbare Hypothese: Durch gezielte Arbeit an bildungssprachlichen Elementen (z.B. Nominalisierungen, komplexe Partizipialkonstruktionen, Passivkonstruktionen o.ä.) und eine Bewusstmachung ihrer konzeptuellen Logik lassen sich gezielt die fachlich angestrebten Kognitionen (Abstrahieren, Fokusverschiebung vom Subjektiven zum Systemischen etc.) auslösen und trainieren - und damit durch Spracharbeit gezielt fachliche Lernziele verfolgen.

\section{KL-Forschungsfrage Beispiel 2}

Theoretisch motivierte Annahme: Theoretisch durch die Kognitive Linguistik gestützt ist die Grundannahme, dass konzeptuelle Metaphern ein Grundprinzip menschlicher Wissensrepräsentation und -verarbeitung darstellen, das sich in unseren Denkmustern, aber auch in der sprachlichen Struktur widerspiegelt. Fachdiskurse folgen z.T. durchgängigen metaphorischen Feldern (z.B. wird eine

Barbara Hinger (Hg.), Zweite „Tagung der Fachdidaktik“ 2015.

Sprachsensibler Sach-Fach-Unterricht - Sprachen im Sprachunterricht.

(C) 2016 innsbruck university press, ISBN 978-3-903122-51-2, DOI 10.15203/3122-51-2 
wissenschaftsnahe Vorstellung konzeptuell metaphorisiert als „Gebäude“ - daraus ergeben sich ein ganzes Wort- und Phrasenfeld: „Die Basis für diese Argumentation“; „die grundlegende These“; „eine wenig fundierte Vorstellung“; „eine Argumentation aufbauen“; ,eine instabile Argumentation“ u.v.m.) Um solche Strukturen zu meistern, ist eine Bewusstmachung der unterliegenden Systematik hilfreich.

Überprüfbare Hypothese: Das Bewusstmachen der dem Fachdiskurs unterliegenden Metaphorik und der radialen Ausweitung in damit verbundenen Konzeptualisierungen führt zur effizienteren Textkompetenzförderung im Fach als reines Auswendiglernen von Phrasen ohne einen aufgezeigten unterliegenden Sinnzusammenhang.

\section{KL-Forschungsfrage Beispiel 3:}

Theoretisch motivierte Annahme: Ein kognitiv-linguistischer Theorierahmen impliziert, dass Personen mentale Scripts und assoziative Wissensstrukturen anhand von prototypischen Beispielen und durch bedeutungshaltigen Gebrauch aufbauen. Fachlernen und Sprachlernen basieren dabei prinzipiell auf denselben Prinzipien.

Überprüfbare Hypothese: Sprachfördermaßnahmen werden erfolgreicher sein, wenn sie an prototypische fachliche Aktivitäten gekoppelt sind und ihre Bedeutung für die Fachlichkeit transparent gemacht wird, als wenn sie sprachformfokussiert ohne Bezug auf die fachliche Aussage vorgenommen werden.

Wie hier deutlich geworden sein sollte, ermöglicht eine Verbindung von soziokulturell und kognitiv-linguistisch motivierten Annahmen eine Fokussierung der Verbindung von integrativem Fach- und Sprachlernen in unterschiedlichen Auflösungsgraden, die dennoch ein stimmiges Ganzes ergibt. Damit wird es möglich, durch den Einbezug von kognitiv-linguistischen Ansätzen andere theoretisch hergeleitete hypothesentestende Untersuchungsfragen zu stellen als durch soziofunktionale Theorien allein, wobei aber gleichzeitig eine Stringenz mit soziofunktionalen Konstrukten auf höherer Ebene beibehalten wird. Verbindet 
man zum Beispiel die Soziokulturelle Theorie mit der Kognitiven Linguistik, so liefert diese mit ihren konkreten Annahmen über z.B. die bekannte Rolle von Interaktion, der Zone of Proximal Development und der Mediation (s. im Detail z.B. Lantolf \& Poehner 2014) andere Konstrukte als jene mit ihrem Ausgangspunkt in Frames und mentalen Modellen, konzeptueller Metapher, radialen Kategorien, Prototypensemantik sowie ihren Annahmen über embodied cognition (s. zur Erläuterung im Einzelnen z.B. Evans \& Green 2006). Auf der Basis der Verknüpfung beider Theorien wäre es möglich, ein gut verankertes Programm für die Erforschung von integrativem Fach- und Sprachlernen aufzustellen.

\section{Zusammenfassung und Fazit}

In den obigen Ausführungen habe ich zunächst dafür plädiert, dass eine wissenschaftlich solide Didaktik integrativen Fach- und Sprachlernens ein stringentes und empirisch valides theoretisches Modell erfordert, das beide Lerndimensionen abbilden kann. Dabei habe ich einen Vorteil für das Sachfachlernen durch Spracharbeit vermutet. Ich habe argumentiert, dass ein Nachweis einer solchen Beziehung eine explizite Theoriegrundlage erfordert, und daher die in den bisherigen Diskursen zu integrativem Fach- und Sprachlernen verwendeten sozio-funktionalen Theorierahmen mit dem Ergebnis besprochen, dass sie dazu geeignet sind, genügend Raum für die integrale Abbildung von gleichzeitigem Fach- und Sprachenlernen bereitzustellen. Abschließend habe ich mich dem derzeit aufkommenden Trend in diesen Theorien angeschlossen, der die Kognitive Linguistik als hilfreichen Theorierahmen auf kognitiver Mikroebene identifiziert.

Wissenschaftlich solide didaktische Forschung braucht Theorien; allgemeine Lerntheorien, aber auch Spracherwerbstheorien. Der R. Ellis zugeschriebene Ausspruch „,nothing is as practical as a good theory“ trifft meines Erachtens vollends zu. Ein solides Theoriekonstrukt ist die Grundlage u.a. für Wirkungsforschung. Der Bereich des integrativen Fach- und Sprachlernens kann dabei auf eine gut vorbereitete und empirisch gut etablierte Theorielandschaft zurückgreifen; es gilt, sie ausreichend zu rezipieren, zu erschließen und entsprechende empirisch ausgerichtete Forschungsarbeiten zu verwirklichen.

Barbara Hinger (Hg.), Zweite „Tagung der Fachdidaktik“ 2015.

Sprachsensibler Sach-Fach-Unterricht - Sprachen im Sprachunterricht.

(C) 2016 innsbruck university press, ISBN 978-3-903122-51-2, DOI 10.15203/3122-51-2 


\section{Literatur}

Baumann, B. \& Becker-Mrotzek, M. (2014). Sprachförderung und Deutsch als Zweitsprache an deutschen Schulen: Was leistet die Lebrerbildung? Überblick, Analysen und Handlungsempfeblungen. Köln: Mercator-Institut für Sprachförderung und Deutsch als Zweitsprache.

Bazerman, C. (2009). Genre and Cognitive Development: Beyond Writing to Learn. In C. Bazerman, A. Bonini \& D. Figueiredo (Hrsg.), Genre in a Changing World (279-294). West Lafayette: Parlor Press.

Bonnet, A. (2004). Chemie im Bilingualen Unterricht: Kompetenzerwerb durch Interaktion. Opladen: Leske und Budrich.

Breidbach, S. (2007). Bildung, Kultur, Wissenschaft. Reflexive Didaktik. für den bilingualen Sachfachunterricht. Münster: Waxmann.

Breidbach, S. \& Viebrock, B. (2012). CLIL in Germany: Results from Recent Research in a Contested Field of Education. International CLIL Research Journal, 1 (4), 5-16. Verfügbar unter: http://www.icrj.eu/14/article1.html [29.03.2016].

Budde, M. \& Michalak, M. (2015). Sprachenfächer und ihr Beitrag zur fachsprachlichen Förderung. In M. Michalak (Hrsg.), Sprache als Lernmedium im Fachunterricht. Theorien und Modelle für das sprachbewusste Lebren und Lernen (9-33). Baltmannsweiler: Schneider Verlag Hohengehren.

Bundesinstitut bifie (2011). Kompeten₹modell Naturwissenschaften 8. Schulstufe. Verfügbar unter: https:// www.bifie.at/system/files/dl/bist_nawi_kompetenzmodell-8_2011-10-21.pdf [29.03.2016].

Busch, H. \& Ralle, B. (2013). Fachsprachliche Kompetenzen in heterogenen Lerngruppen fördern. In S. Bernholt (Hrsg.), Inquiry-based Learning - Forschendes Lernen. Gesellschaft für Didaktik der Chemie und Physik, Jahrestagung in Hannover 2012 (389-391). Kiel: Leibniz-Institut für die Pädagogik der Naturwissenschaften und Mathematik.

Butler, C. (2013). Systemic Functional Linguistics, Cognitive Linguistics and psycholinguistics: Opportunities for dialogue. Functions of Language, 20 (2), 185-218.

Coffin, C. \& Donohue, J. P. (2012). Academic Literacies and systemic functional linguistics: How do they relate? Journal of English for Academic Purposes, 11, 64-75.

Coyle, D., Hood, P. \& Marsh, D. (2010). CLIL: Content and Language Integrated Learning. Cambridge: Cambridge University Press.

Dalton-Puffer, C. (2013). A construct of cognitive discourse functions for conceptualising contentlanguage integration in CLIL and multilingual education. EuJAL, 1 (2), 216-253.

Barbara Hinger (Hg.), Zweite „Tagung der Fachdidaktik“ 2015.

Sprachsensibler Sach-Fach-Unterricht - Sprachen im Sprachunterricht.

(C) 2016 innsbruck university press, ISBN 978-3-903122-51-2, DOI 10.15203/3122-51-2 
Ellis, R. \& Shintani, N. (2014). Exploring Second Language Pedagogy through Second Language Acquisition Research. Abingdon: Routledge.

Evans, V. \& Green, M. (2006). Cognitive Linguistics. An Introduction. Edinburgh: Edinburgh University Press. Davydov, V.V. (2004). Problems of developmental instruction. A theoretical and experimental psychological study. Moskau: Akademiya Press.

Duff, P. A. \& Talmy, S. (2011). Language Socialization Approaches to Second Language Acquisition: Social, cultural, and linguistic development in additional languages. In D. Atkinson (Hrsg.), Alternative Approaches to Second Language Acquisition (95-116). London, New York: Routledge.

Fawcett, R. (2000). A theory of syntax for systemic functional linguistics. Amsterdam, Philadelphia: Benjamins.

Ehlich, K. \& Rehbein, J. (1983) (Hrsg.). Kommunikation in Scbule und Hochschule. Linguistische und ethnomethodologische Analysen. Tübingen: Narr.

Hallet, W. (1998). The Bilingual Triangle. Überlegungen zu einer Didaktik des bilingualen Sachfachunterrichts. Praxis des neusprachlichen Unterrichts, 45 (2), 115-125.

Halliday, M. A. K. \& Martin, J. R. (1993). Writing Science: Literacy and discursive power. Pittsburgh, PA: University of Pittsburgh Press.

Halliday, M. A. K. \& Matthiessen, C. M. I. M. (2004). An Introduction to Functional Grammar (3. Aufl.). London: Hodder Arnold.

Handro, S. \& Schönemann, B. (Hrsg.) (2010). Geschichte und Sprache. Berlin: LIT-Verlag.

Heine, L. (2010). Problem-solving in a foreign language. A study in Content and Language Integrated Learning. Berlin, New York: De Gruyter.

Heine, L. (2013). Empirische Erforschung des Bilingualen Unterrichts. In W. Hallet \& F. G. Königs (Hrsg.), Handbuch Bilingualer Unterricht/Content and Language Integrated Learning (216-221). Seelze: Klett Kallmeyer.

Heine, L. (2015). Sprachtheorien und ihre Explizierung in der unterrichtsbezogenen Fremdsprachenforschung. In A. Grünewald \& S. Doff (Hrsg.), WECHSEL-Jahre? Wandel und Wirken in der Fremdsprachenforschung (177-192). Trier: wvt.

Heine, L. (i.Dr.). Lernziele in CLIL. Zeitschrift für Interkulturellen Fremdsprachenunterricht.

Kircher, E., Girwidz, R. \& Häußler, P. (Hrsg.) (2014). Physikdidaktik: Theorie und Praxis. Berlin: Springer.

KMK (2015): Bildungsstandards. Verfügbar unter: http://www.icri.eu/14/article1.html [29.03.2016]. Krüger, D. \& Vogt, H. (2007). Theorien in der biologiedidaktischen Forschung. Ein Handbuch für Lehramtsstudenten und Doktoranden. Berlin et al.: Springer.

Barbara Hinger (Hg.), Zweite „Tagung der Fachdidaktik“ 2015.

Sprachsensibler Sach-Fach-Unterricht - Sprachen im Sprachunterricht.

(C) 2016 innsbruck university press, ISBN 978-3-903122-51-2, DOI 10.15203/3122-51-2 
Lantolf, J. P., Thorne, S. L. \& Poehner, M. E. (2015). Sociocultural Theory and Second Language Development. In B. VanPatten \& J. Williams (Hrsg.), Theories in Second Language Acquisition. An Introduction (207-226). New York: Routledge.

Lantolf, J. P. \& Poehner, M. E. (2014). Sociocultural Theory and the Pedagogical Imperative in L2 Education. New York: Routledge.

Long, M. \& Robinson, P. (1998). Focus on form: Theory, research, and practice. In C. Doughty \& J. Williams (Hrsg.), Focus on form in classroom second language acquisition (15-63). Cambridge: Cambridge University Press.

Lillis, T. \& Scott, M. (2008). Defining academic literacies research: issues of epistemology, ideology and strategy. Journal of Applied Linguistics, 4 (1), 5-32.

Prediger, S. (2013). Darstellungen, Register und mentale Konstruktion von Bedeutungen und Beziehungen - Mathematikspezifische sprachliche Herausforderungen identifizieren und überwinden. In M. Becker-Mrotzek, K. Schramm, E. Thürmann \& H. J. Vollmer (Hrsg.), Sprache im Fach - Sprachlichkeit und facbliches Lernen (167-183). Münster et al.: Waxmann.

Rotter, D. (2015). Der Focus-on-Form-Ansatz in der Sprachförderung. Eine empirische Untersuchung der Lehrer-Lerner-Interaktion im DaZ-Grundschulkontext. Münster: Waxmann.

Schleppegrell, M. (2004). The Language of Schooling. A Functional Linguistics Perspective. Mahwah: Erlbaum.

Swain, M., Lapkin, S., Knouzi, I., Suzuki, W. \& Brooks, L. (2009). Languaging: University students learn the grammatical concept of voice in French. Modern Language Journal, 93, 5-29.

Thesen, L. \& Pletzen, E. V. (Hrsg.) (2006). Academic literacy and the languages of change. London: Continuum

Voss, B. (2012). Fremdsprachenunterricht an Hochschulen: UNIcert und die Hochschulspezifik. In T. Tinnefeld (Hrsg.), Hochschulischer Fremdsprachenunterricht: Anforderungen - Ausrichtung - Speaifike (359-368). Saarbrücken: htw.

Weigand, E. (1992): A Case for an Integrating Procedure of Theoretical Reflection and Empirical Analysis. In S. Stati \& E. Weigand (Hrsg.), Methodologie der Dialoganalyse (57-64). Tübingen: Niemeyer.

Wygotski, L. (1987). Ausgewäblte Schriften. Band 2: Arbeiten zur psychischen Entwicklung der Persönlichkeit. Köln: Pahl-Rugenstein.

Zydatiß, W. (2007). Deutsch-Englische Züge in Berlin (DEZIBEL). Eine Evaluation des bilingualen Sachfachunterrichts an Gymnasien. Kontext, Kompetenzen, Konsequenzen. Frankfurt a. M.: Peter Lang.

Barbara Hinger (Hg.), Zweite „Tagung der Fachdidaktik“ 2015.

Sprachsensibler Sach-Fach-Unterricht - Sprachen im Sprachunterricht.

(C) 2016 innsbruck university press, ISBN 978-3-903122-51-2, DOI 10.15203/3122-51-2 
